\title{
Anaplastic large cell neuroblastoma and lymphoma- cytological twins
}

\begin{abstract}
Anaplastic large cell neuroblastomas (ALCNB) are a subset of undifferentiated neuroblastomas with marked pleomorphic and anaplastic cytomorphological features which are characterized by the absence or marked paucity of histological clues for the diagnosis of neuroblastoma that render them diagnostically challenging. Here in, we are presenting a case of ALCNB in which the clinical, radiological and cytological features were misleading to a diagnosis of lymphoma. High degree of cytological suspicion, especially when an abdominal mass with internal calcification presents in children less than 5 years of age in the absence of classical cytological picture of neuroblastoma like resetting and neuropil but presence of nuclear anaplasia with many bizarre and multinucleated cells mimicking lymphoma, might help in delineating these cytological twins.
\end{abstract}

Further study of this variant of neuroblastoma is required as it poses a diagnostic dilemma, due to the presence of similar types of cells as that of anaplastic large cell lymphoma(ALCL). It not only elucidates the prognosis but also aids in establishing an appropriate differential diagnosis and determine the optimal therapy.

Keywords: anaplastic large cell neuroblastoma, anaplastic large cell lymphoma
Volume 8 Issue 3 - 2018

\author{
Mona Bargotya, Kiran Aggarwal, Archna \\ Rautela Pahwa, Nitin Sood \\ University Lady Hardinge Medical College, India
}

Correspondence: Mona Bargotya, Senior Resident, University Lady Hardinge Medical College, India, Tel 009I 9953I 696I I, Email drmona2208@gmail.com

Received: April 26, 2018 | Published: May 08, 2018

\section{Introduction}

Neuroblastoma, one of the major categories of peripheral neuroblastic tumors, has been described as a small, blue, monomorphic, round cell tumor of childhood. However, these actually are far more heterogeneous morphologically and biologically than is generally believed. Anaplastic large cell neuroblastoma (ALCNB) is a striking aspect of neuroblastoma and is a very rare entity which was first described by Cozzutto \& Carbone ${ }^{1}$ in $1988 .{ }^{1}$ ALCNB is a rare subset of undifferentiated neuroblastomas which presents with marked pleomorphic and anaplastic features as well as presence of bizarre and monstrous cells which are the hallmark of this neuroblastoma variant, that render them diagnostically challenging. ${ }^{1-3}$ Further, ALCNB presenting with peripheral lymphadenopathy and ascites is very uncommon. Herein, we discuss a case of retroperitoneal ALCNB in a 3 years old female presenting with supraclavicular lymphadenopathy and ascites, which posed a diagnostic dilemma due to its unusual clinical presentation and cytomorphological features.

\section{Case summary}

A 3 years old female child presented with complaints of high grade fever, abdominal mass and a left supraclavicular lymph node since 2 months. On examination, single left supraclavicular lymph node measuring $1 \times 0.5 \mathrm{~cm}$ and distended abdomen with free fluid was observed. Ultrasonography of abdomen revealed a large, lobulated, heteroechoic, conglomerated nodal mass of size $11.3 \times 8.3 \times 7 \mathrm{~cm}$ in retro peritoneum with multiple anechoic areas and ascites. On this basis, a clinical diagnosis of Non-Hodgkin's lymphoma was made. FNAC performed from abdominal mass and lymph node showed similar cytomorphological features. Smears were cellular, showing single cells as well as poorly cohesive groups of large anaplastic cells with large, pleomorphic vesicular nuclei with 2 to 3 prominent nucleoli and scant to moderate amount of cytoplasm (Figure 1). Many binucleated and multinucleated tumor cells with presence of doughnut, embryoid, reniform and occasional wreath cells were identified (Figure 2). Many atypical mitotic figures were also seen. Based on these cytological features, a diagnosis of anaplastic large cell lymphoma was suggested. Meanwhile CT scan revealed a heterogenously enhancing retroperitoneal nodal mass measuring $11 \times 8 \times 7 \mathrm{~cm}$ with encasement of major vessels \& bowel along with single foci of calcification and chest involvement suggestive of lymphoma/neuroblastoma (Figure 3). Serum VMA level was also found to be raised (17.5mg/24hrs). Following this lymph node biopsy was done and the histopathology showed complete loss of architecture \& replacement by tumor .The tumor cells were arranged in nests and cords separated by fibrous septae with occasional rosettes and absence of neuropil (Figure 4). The tumor cells were large, round to oval in shape with round to oval nucleus with moderate anisonucleosis, irregular nuclear membrane, coarsely clumped chromatin, 2 to 3 prominent nucleoli and scant to moderate amount of eosinophilic cytoplasm. The tumor cells show similar cytomorphological features as in FNAC smears and many atypical mitotic figures $(03 / \mathrm{Hpf})$ were seen. Immunohistochemical panel for LCA, CD15, CD30, ALK, SMA, synaptophysin, chromogranin and NSE was applied. The present case showed strong positivity for chromogranin and NSE, however was negative for all the other markers (Figure 5).

On the basis of these histological, immunological and radiological findings a diagnosis of metastatic an aplastic large cell neuroblastoma was made and the patient was immediately put on chemotherapy. After 2 cycles of chemotherapy, the size of the mass decreased significantly along with the reduction of VMA levels and the patient responded very well to therapy with improvement of her general condition. Post chemotherapy period has been uneventful till date. 


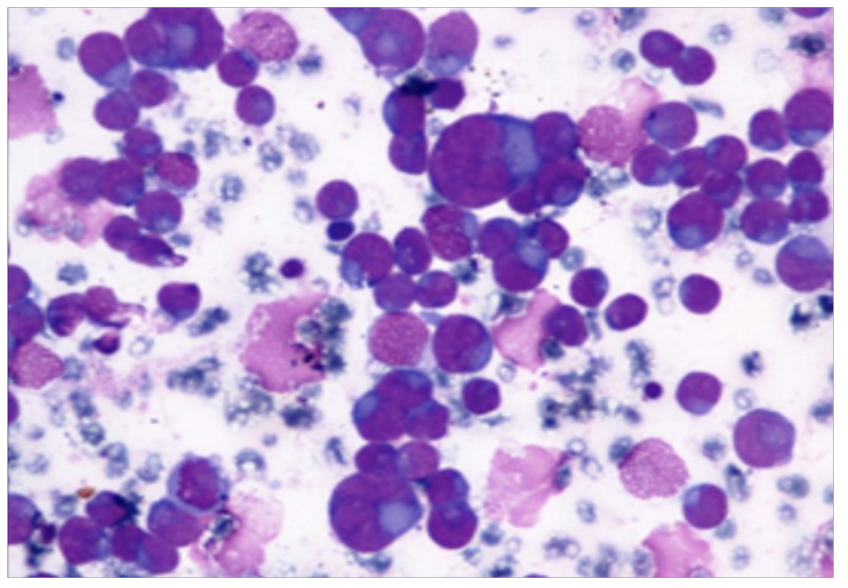

Figure I FNAC smear showing single cells and poorly cohesive groups of anaplastic cells with moderate amount of cytoplasm and large, pleomorphic vescicular nuclei with 2-3 prominent nucleoli (Giemsa x400).
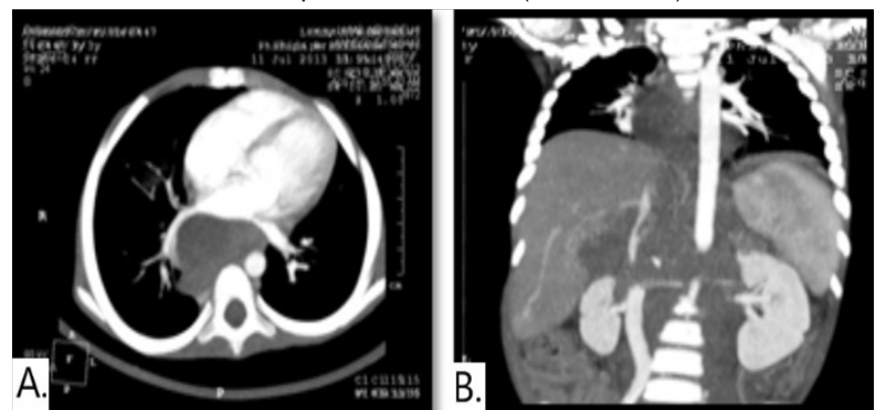

Figure 2 CT-scan showing heterogenously enhancing retroperitoneal nodal mass measuring $1 \mathrm{I} \times 8 \times 7 \mathrm{~cm}$ with encasement of major vessels and bowel, single foci of calcification with chest involvement.
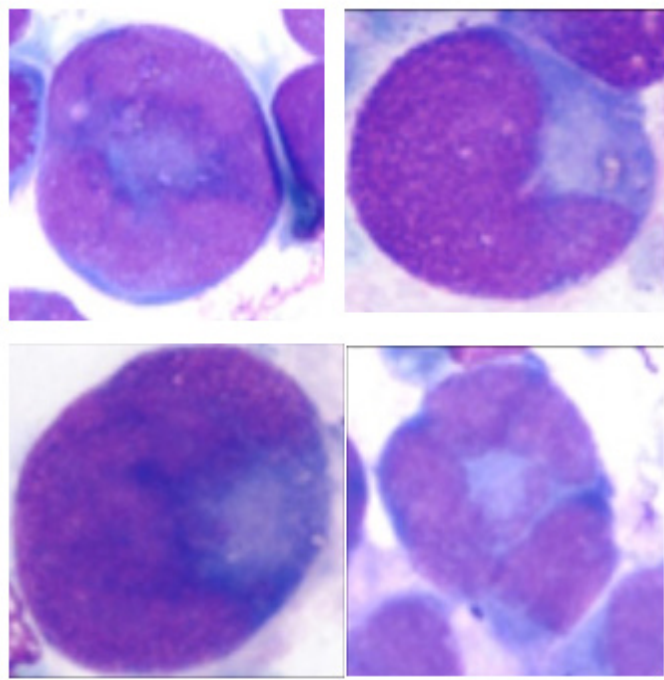

Figure 3 Different cytomorpholgical forms of tumor cells: a) doughnut cell, b) reniform cell, c)embryoid cell\& d) wreath cell (Giemsal000x).

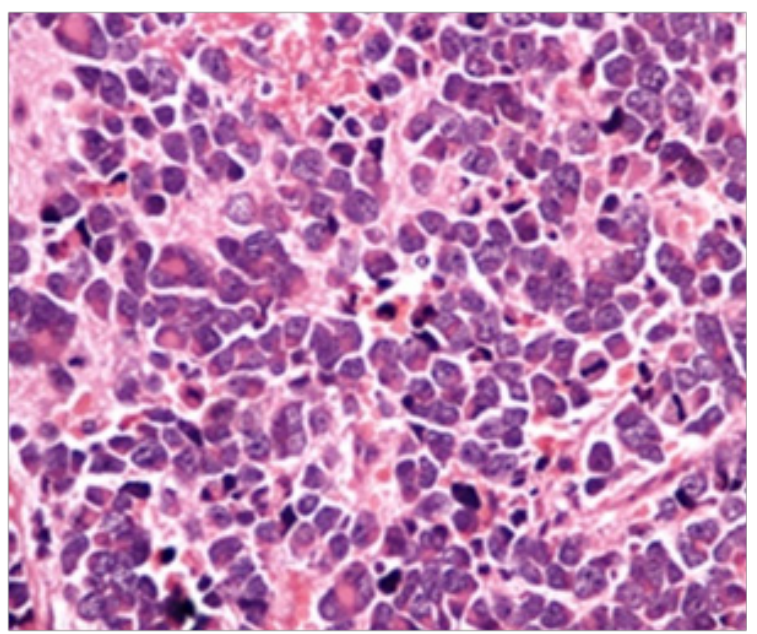

Figure 4 Tumor cells arranged in nests and cords separated by fibrous septae with absence of neuropil (H\&E400x).
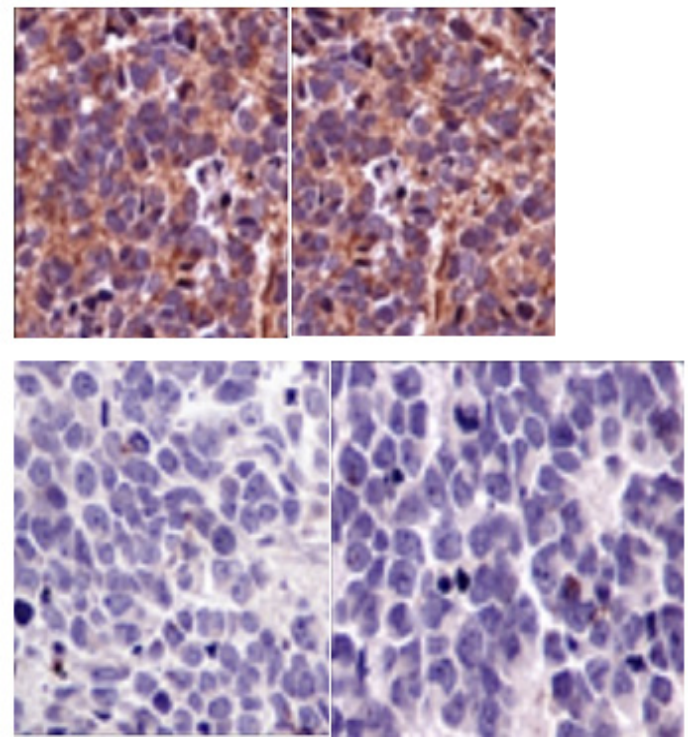

Figure 5 Tumor cells showing strong chromogranin (A) and NSE (B) positivity and CD30 (C)and ALK(D) negativity.

\section{Discussion}

Based on its unique histologic phenotype (large, pleomorphic and anaplastic cells with sharp nuclear outlines and prominent nucleoli) ALCNB is recognized as a new, distinct entity within the neuroblastoma category. ALCNB is included within the undifferentiated and poorly differentiated subtypes of neuroblastoma (Schwannian stroma poor) category, following the guidelines of the new International Neuroblastoma Pathology Classification(INPC 1999). ${ }^{4,5}$ It is characterized by striking degree of cellular and nuclear pleomorphism and anaplasia with bizarre and monstrous morphology along with a high mitosis-karyorrhex is index and a low incidence of calcification. It responds poorly to chemotherapy and has unfavorable prognosis with aggressive clinical behavior. ${ }^{6,7}$ 
ALCL which has been recognized as a distinct heterogeneous clinicopathologic entity that may have a T-cell, B-cell, or indeterminate (null) phenotype and may or may not express the anaplastic lymphoma kinase (ALK). ${ }^{8}$ It is characterized by proliferation of large pleomorphic and anaplastic lymphoid cells which strongly expresses CD30 antigen and usually grows in a cohesive pattern and have a propensity to involve lymph node sinuses.

\section{Conclusion}

To the best of our knowledge this is the first case of ALCNB presenting with lymphadenopathy and ascites. It posed a diagnostic dilemma due to presence of similar morphology as that of ALCL. Hence, it is recommended that further investigation of larger series of peripheral neuroblastic tumors in national and international studies is required for the confirmation of the existence of this rare and distinct subset of neuroblatoma with unfavorable clinical outcome. Independent of prognosis, the definition of ALCNB is a useful conceptual specification and aids in differential diagnosis. ${ }^{9}$

\section{Acknowledgments}

None.

\section{Conflict of interest}

The author declares there is no conflict of interest.

\section{References}

1. Cuzzutto C, Carbone A. Pleomorphic (Anaplastic) neuroblastoma. Arch Pathol Lab Med.1998;112(6):621-625.

2. Christopher DM Fletcher. Diagnostic Histopathology of Tumors. Elsevier Saunders. 2013. p. 2074-2080.

3. Juan Rosai. Rosai and Ackerman's Surgical Pathology. Elsevier Mosby. 2011. p.1068-73.

4. Shimada H, Ambros IM, Dehner LP, et al. The International Neuroblastoma Pathology Classification (the Shimada System). Cancer. 1999;86(2):364 372.

5. Shimada H, Ambros IM, Dehner LP, et al. Terminology and morphology criteria of neuroblastic tumors. Recommendations by the International Neuroblastoma Pathology Committee. Cancer. 1999;86(2):349-363.

6. Chatten J. anaplastic neuroblastoma. Letter to the editor. Arch Pathol Lab Med. 1989;113:9-10.

7. Tornoczky T, Kalman E, Kajtar PG, et al. Large cell neuroblastoma. A distinctive phenotype of neuroblastoma with aggressive clinical behavior. Cancer. 2004;100(2):390-7.

8. Kinney MC, Kadin ME. The pathologic and clinical spectrum of anaplastic large cell lymphoma and correlation with ALK gene dysregulation. Am J Clin Pathol. 1999;111(Suppl 1):S56-S57.

9. Dehner LP. Anaplasia in solid malignant tumors of childhood. Arch Pathol Lad Med.1989;113(1):11-12. 